\title{
Simplex-Tree Based Kinematics of Foldable Objects as Multi-body Systems Involving Loops
}

\author{
Li Han and Lee Rudolph \\ Department of Mathematics and Computer Science \\ Clark University, Worcester, MA 01610 \\ Email: lhan@clarku.edu, lrudolph@black.clarku.edu
}

\begin{abstract}
Many practical multi-body systems involve loops. Studying the kinematics of such systems has been challenging, partly because of the requirement of maintaining loop closure constraints, which have conventionally been formulated as highly nonlinear equations in joint parameters. Recently, novel parameters defined by trees of triangles have been introduced for a broad class of linkage systems involving loops (e.g., spatial loops with spherical joints and planar loops with revolute joints); these parameters greatly simplify kinematics related computations and endow system configuration spaces with highly tractable piecewise convex geometries. In this paper, we describe a more general approach for multi-body systems, with loops, that allow construction trees of simplices. We illustrate the applicability and efficiency of our simplex-tree based approach to kinematics by a study of foldable objects. We present two sets of new parameters for single-vertex rigid fold kinematics; like the parameters in the triangle-tree prototype, each has a geometrically meaningful and computationally tractable constraint formulation, and each endows the configuration space with a nice geometry.
\end{abstract}

\section{INTRODUCTION}

Many practical multi-body systems involve one or more loops_-physical (in parallel platforms, ring-type molecules, ...) or virtual (in inverse kinematics of serial manipulators or molecular chains,...). The kinematics of loop systems is complicated by the so-called loop closure constraint, i.e., the need to maintain the closed chain structure (see e.g. books [1, $2,3,4]$ and references therein). There are many other practical issues, like joint limits (and other system limits) and collision avoidance, but here we focus on loop closure to the exclusion of all other constraints. In general, progress in any subset of system related issues contributes to progress in overall system knowledge; and the loop closure constraint is a recognized stumbling block in the study of multi-body loop systems.

The main difficulty with loop systems is the generally complex constraint formulation with respect to system parameters. To date, the most widely used linkage parameters are joint parameters, such as joint angles for rotational joints and linear displacements for prismatic joints. Conventionally, loop closure constraints have been formulated as equality constraints (of highly non-linear functions) over joint parameters. This formulation shows that for generic linkages the set of closure configurations is a smooth submanifold of the ambient joint parameter space, and in many cases its topology is partly or completely known (see, e.g., [5, 6] and [7, 8], which treat planar and spatial linkages with spherical-type joints).

Patent pending.
Smooth manifolds are characterized by the existence of local coordinates, but although some manifolds are equipped with global coordinates (like the joint angle parameters, in the cases of a serial chain without closure constraints or $[7,8]$ a planar loop satisfying the technical " 3 long links" condition), typically a manifold has neither global coordinates nor any standard atlas of local coordinate charts. Thus, calculations that depend on coordinates are often difficult to perform.

Some recent kinematic work uses new parameters that are not conventional joint parameters. One series of papers [9, $10,11,12,13]$ presented novel formulations and techniques, including distance geometry, linear programming and flag manifolds, to solve inverse kinematics, identify trilaterable 6DOF parallel and serial manipulators, and parametrize configurations of flag manipulators. Loosely speaking, a system is "trilaterable" as addressed there if it can be decomposed into tetrahedra in such a way that all unknown edge-lengths of the tetrahedra can be systematically computed from known edge-lengths using distance constraints (triangle inequalities and Cayley-Menger constraints; see below). In those papers, the trilaterable systems to be solved are already given in trilaterable form: the kinematic structure explicitly includes all distance parameters needed to determine system configurations (e.g., lengths of the legs between base and platform of a parallel manipulator). We believe those papers were the first to recognize and utilize trilaterability of the systems they discuss.

In papers [14, 15, 16], we presented a different set of parameters for a class of linkages including planar loops with revolute joints and spatial loops with spherical joints. Our parameters are diagonal lengths (inter-joint distances) and triangle orientation parameters (discrete signs in the plane, dihedral angles in space). In essence, to define the parameters one joint of each loop is used as an anchor; diagonals are drawn from it to all non-adjacent joints, partitioning the loop into an open chain of triangles. The diagonal lengths and triangle orientation parameters are precisely enough to determine the shapes and relative configurations of these anchored triangles, which in turn determine the loop configurations. We proved that the defined parameters are indeed coordinates on the set of closure configurations. Further, we observed that the resulting atlas of local coordinate charts endows that space with a nice geometric structure we called "practical convexity", and remarked that our approach generalizes to any linkage system that can be decomposed into a tree of triangles. 


\section{Novel Idea: Construction Trees of Simplices}

Here we will present a general simplex-tree based parametrization approach for multi-body systems allowing construction trees of simplices; it includes the triangle-based approach of $[14,15]$ as a special case. Due to space limits, we write this paper somewhat intuitively and informally.

Before describing our new parametrization, we make a few comments. First, following the approach in [14, 15], we will focus on multi-body systems' deformations, that is, configurations with rigid motions factored out. The set of all deformations, called the deformation space, is mathematically the quotient space of the configuration space modulo the group of rigid motions that respect system constraints: DSpace $=$ CSpace/RM.

Second, we note that many multi-body systems can be studied as multi-point systems, e.g., by reducing each rigid member in the system to at most 4 general points in the member. Further, there may exist distance constraints among the points, which can be modeled as links between points: each pair of points subject to distance constraints can be modeled by a link that joins them, of fixed or variable length depending on the nature of the constraints. By such means, we can use linkage concepts, terms, and notations to study multi-object and multi-point systems under distance constraints.

Last, we recall some basic definitions concerning simplices, and facts about their geometry. In this paper we need only simplices of dimension at most 3: a 0 -simplex is a point; a 1 -simplex is a line segment; a 2 -simplex is a triangle; a 3 simplex is a tetrahedron. A 0 -dimensional face of a simplex is called a vertex, and a 1-dimensional face is called an edge. For $k \geq 2$, the edge lengths (i.e., inter-vertex distances) of a $k$ simplex are subject to non-trivial constraints; the most familiar and simplest are the "triangle inequality" constraints for $k=$ 2 , and, in the Euclidean case, non-negativity of its CayleyMenger determinant [17] (see Eq. (2) below) for all $k$. If all edge lengths of a simplex $\sigma$ are fixed, then the shape of $\sigma$ is essentially fixed. In the language of deformations, all points of $\operatorname{DSpace}(\sigma)$ are isolated; e.g., in Euclidean space, DSpace $(\sigma)$ contains just 1 or 2 points, the latter case happening only for deformations of an $n$-simplex in $\mathbb{R}^{n}$ (its 2 deformations are distinguished by their orientation).

Definition. Our new approach to kinematics of linkage systems involving loops (and other multi-point systems under distance constraints) is based on representing the system under study by a construction tree of simplices. We say a tree of simplices is a construction tree of a given linkage system if the simplices satisfy the following three conditions. (1) Each link in the linkage system is an edge of at least one simplex in the tree. (2) The set of points of the multi-point linkage system equals the set of all vertices of all simplices in the tree. (3) The deformations of the linkage system can be constructed from the shapes of the simplices and relative configurations of simplices adjacent in the tree.

Simplex Placement Procedure and Deformation Construction. We can use a tree traversal process to construct any deformation of a multi-point system with a construction tree $\mathcal{T}=(V(T), E(T))$ of simplices, given the following necessary and sufficient data about the deformation: (i) for each node $\sigma \in V(\mathcal{T})$, the shape of the simplex $\sigma$, and (ii) for each edge $\{\sigma, \tau\} \in E(\mathcal{T})$, the relative configuration of the simplices $\sigma$ and $\tau$ in the ambient space $\mathbb{R}^{n}$. Indeed, placing a simplex in an ambient space is equivalent to determining the coordinates of its vertices. Now, given the data (i) and (ii), we construct the corresponding deformation of the multi-point system recursively as follows. (I) Place any simplex $\sigma \in V(\mathcal{T})$ anywhere in its ambient space (in case $\operatorname{dim}(\sigma)$ equals the ambient dimension, an orientation parameter specifies one of its two orientations). (II) If for some edge $\{\rho, \tau\} \in E(\mathcal{T})$, the simplex $\rho$ has already been placed in space but the simplex $\tau$ has not yet been placed, then use the data (ii) to place $\tau$. When this simplex placement procedure terminates, the deformation has been constructed.

New Parameters. Two types of parameters hold the deformation data (i) and (ii): shape parameters (associated to nodes of $\mathcal{T}$ ) and orientation parameters (associated to edges of $\mathcal{T}$ ).

The shape of a simplex is determined by its edge lengths. In any given simplex in $V(\mathcal{T})$, some edges may be links (some with fixed lengths and others with variable lengths); we call a construction tree simplex edge a diagonal of the linkage if it is not a link. Our shape parameters for a linkage comprise the lengths of all variable edges and diagonals from the tree.

Two adjacent simplices in a construction tree share a subsimplex (e.g., two triangles with a common edge). Thus there is no relative translation between two adjacent simplices, only at most a relative reorientation (about the common subsimplex)-in essence, an element of that subgroup of the orthogonal group of the ambient Euclidean space which acts as the identity on a linear subspace having the dimension of the common sub-simplex. Our orientation parameters for a linkage comprise relative reorientation data for adjacent simplices.

In summary, our simplex-based parameters for deformations comprise (a) lengths of diagonals and links of variable length (to give shapes of simplices), and (b) orientation parameters (to give relative configurations of adjacent simplices).

Results. The aforementioned simplex placement procedure for deformation determination indicates how to use simplexbased parameters to completely determine system deformations.

If we define the forward kinematics $(F K)$ of a multi-point system as the determination of system point positions from given parameter values, the procedure is an algorithm that solves the $F K$ problem. Conversely, the inverse kinematics $(I K)$ problem of determining valid simplex-based parameters that satisfy loop closure constraints is equivalent to solving the system deformation space in those parameters.

This approach is very general and applies in many ambient geometries. In this paper, limited to simplices in Euclidean space or the 2-sphere, our main results are as follows.

Theorem 1: Consider a multi-point (or multi-body) system that allows a construction tree $\mathcal{T}$ of simplices. Then: 
(A) The deformations of the system are described by simplex-based parameters.

(B) The $F K$ problem for the system is solved by a simplex placement procedure, with shapes and relative orientations of simplices directly determined by simplex-based parameters.

(C) The $I K$ problem for the system is solved by giving an explicit description of its deformation space (DSpace) in terms of simplex-based parameters. More precisely, DSpace is essentially the product of DStretch and DFlip, where (1) DStretch comprises shape parameters satisfying explicit, simply evaluated constraints (triangle or Cayley-Menger determinant inequalities, and range inequalities) required for successful simplex formation, and is a convex body, while (2) DFlip comprises relative orientation parameters, and is independent of loop closure constraints.

Proof: If (a) the system under investigation is a planar or spatial linkage with (respectively) revolute or spherical joints, and no links of variable length, (b) $\mathcal{T}$ has no node of valence $\geq 3$ (i.e., is a subdivided interval), and (c) each simplex in $V(\mathcal{T})$ is a triangle in $\mathbb{R}^{2}$ or $\mathbb{R}^{3}$, then we gave detailed proofs of (A), (B), and (C2) (using the relevant versions of our simplex placement procedure) in papers [14] and [15]; the proofs in the general case are entirely analogous.

Essentially the same is true of (C1), with an important technical difference. In our earlier papers, DStretch is a convex polytope, because only triangle inequalities (involving link lengths and shape parameters) are involved, and triangle inequalities are linear. Here, in case $V(\mathcal{T})$ includes one or more tetrahedra, Cayley-Menger inequalities (in link lengths and shape parameters) are involved, and these are non-linearin fact, for tetrahedra they are of total degree at most 3 in the squares of the diagonal lengths (and at most quadratic in the square of any one diagonal length). They are, however, still convex; the proof is an exercise in low-dimensional real algebraic geometry (relying heavily on elementary properties of cubics and quadratics). Thus DStretch need not be a polytope (though it will be as long as all nodes are triangles, even if some are spherical triangles) but it is always a piecewisesmooth semi-algebraic convex body.

As stated, in papers $[14,15]$ we made extensive use of the relevant special cases of Theorem 1. Here, just as in those papers, the general theorem shows that the solution of loop closure constraints can be much more efficient in simplexbased parameters than in conventional joint parameters. This gain in efficiency is due to both the convexity of DStretch and the independence of loop closure from orientation parameters.

In the remainder of this paper, we illustrate the applicability and efficiency of simplex-based parameters by studying foldable objects, especially single-vertex rigid folds, for which we present two sets of new parameters, each with geometricallymeaningful constraint formulations.

\section{FoldABle ObJECTS AND PRIOR WORK}

In our daily life, we encounter such foldable objects as paper bags, umbrellas, and space-station antennas. Normal use of foldable objects involves folding and unfolding, but not cutting

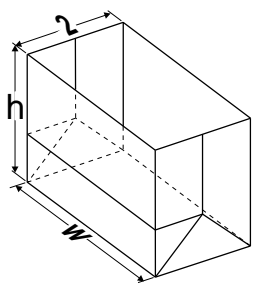

(a) Standard paper bag.

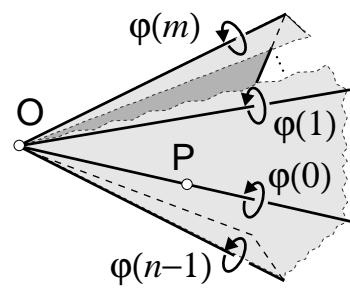

(b) Single-vertex origami.
Fig. 1. Foldable objects.

up; thus many complex constraints are involved in maintaining system structures and allowing system deformations.

Many foldable objects have crease patterns associated with them; see Fig. 1(a) for the creases of a standard paper bag. Each crease line allows relative rotations between two panels sharing the line; it can be viewed as a revolute joint (hinge). Assuming that the panels are rigid, the relative configuration of adjacent panels can be parametrized by an angle that measures their relative rotation about the shared crease line.

Vertices of the crease pattern occur at intersections of crease lines with other crease lines or with the boundary of the object (or both); e.g., the standard bag crease pattern (Fig. 1(a)) has 8 interior vertices and 6 boundary vertices. An interior vertex $O$ on which are incident $n$ crease lines is adjacent to $n$ panels, which surround it in a circuit, or closed chain; if no tearing is allowed this chain has to maintain the closure constraint. In terms of the angle parameters, closure can be understood intuitively as follows: pick any point on any one crease line, and subject that point to a sequence of rotations about each of the crease lines; then the point's final position should coincide with its original position. Mathematically, this becomes (as in [18]) the equation $R\left(v_{0}, \phi_{0}\right) \cdots R\left(v_{n-1}, \phi_{n-1}\right) P=P$, where $P$ denotes a point on a crease line, $v_{i}$ denotes the directional vector for crease line $i$, and $\phi(i)$ denotes the rotation angle about crease line $i$ (see Fig. 1(b)). As this equation must be satisfied for all $P$, it is equivalent to require that the ordered product of the rotation matrices be the identity matrix,

$$
R\left(v_{0}, \phi_{0}\right) \cdots R\left(v_{n-1}, \phi_{n-1}\right)=\mathrm{I} .
$$

Each such equation imposes 3 non-linear constraints on $n$ angular parameters. This means that, for a foldable object with only one non-boundary vertex (i.e., a cycle of $n$ panels surrounding a single common vertex), the space of deformations will generally be of dimension $n-3$. The angular formulation above amounts to studying this deformation space as the subset of the ambient angle space (an $n$-dimensional torus) defined by the constraint (1). Just as for a loop of linear links, the highly non-linear nature of this constraint on the angular parameters makes it technically difficult to understand and compute the structure of DSpace in these traditional coordinates. Foldable objects with more than one non-boundary vertex have yet more complicated descriptions in these parameters. Notwithstanding these difficulties, progress has been made. One interesting result [19] is that DSpace of a paper bag, creased as in Fig. 1(a) and with rigid panels, has isolated points corresponding to 
the folded and completely unfolded states: in this model, a flattened shopping bag cannot be opened.

Kinematic related issues of foldable objects have been studied in various communities. In robotics, in addition to origami folding [18], sheet-metal and carton box folding (e.g. [20, 21, 22, 23]) have also been studied, mainly from the manipulation planning point of view, sometimes with no loop closure constraints for the folded objects. Folding, especially origami folding, has been studied in and outside of scientific communities, and commands a rich literature. The combinatorics and geometry communities have interesting kinematic results on origami folding (see e.g. [24, 25, 26, 27] and references therein), especially on rigid flat origamis, which we use below to give a clear and representative example of our approach. Rigid origamis are modeled to have rigid and planar panels, and flat origami folds are those that can be pressed in a book without (in theory) introducing new creases. Among many interesting results on single-vertex rigid flat origamis, we will use the following properties later (see, e.g., $[24,25]$ for proofs). Assume the cone angles about the vertex add up to $2 \pi$ (as in [27], by cone angle we mean an angle between adjacent crease lines.) Then (a) the number of crease lines, and thus the number of cone angles, for a rigid flat origami vertex is even, and (b) the sum of even-indexed cone angles (thus also the sum of odd-indexed cone angles) is $\pi$.

We also refer readers to a very important and closely related paper [27], where the authors model a single-vertex origami as a spherical polygonal linkage and further prove that all single-vertex origami shapes are reachable from one to another via simple, non-crossing motions. They also consider general conical paper, where the total sum of the cone angles centered at the vertex is not $2 \pi$, and obtain similarly remarkable results for conical paper with certain properties. Their approach relies on natural extensions to the sphere of planar Euclidean rigidity results regarding the existence and combinatorial characterization of expansive motions, which have been used in the recent breakthrough on collision-free convexifying (straightening) Euclidean polygonal loops (open chains) [28, 29]. One of our methods for single vertex origamis also models such a system as a spherical loop. But we use spherical triangles and corresponding parameters to study these loops, and explicitly parametrize their DSpaces, a very different approach from that in [27]. Further comparing results, paper [27] proves that the valid subset of DSpace (i.e., DFree, to use a naming scheme parallel to the well-known CFree for the valid subset of CSpace) is connected, and provides an efficient collision-free path planner; our approach can solve the complete DSpace structure, but does not apply immediately to DFree.

\section{SimpleX-Tree BAsed Kinematics FOR Folds}

Most of this section is devoted to the kinematics of a singlevertex fold, that is, a foldable object with multiple rigid and planar panels incident on one vertex $O$, each panel having two crease lines incident on it at that vertex (see Fig. 1(b)). The rigid panels and crease lines incident on $O$ define a generalized cone with apex at $O$, so $O$ is called the cone apex. Crease lines correspond to edges of the cone, and panels can rotate about crease lines without tearing, so cone deformations are subject to the loop closure constraint if panels and crease lines are to stay intact. Rather than describe DSpace of this cone in terms of angles subject to a constraint of the form (1), we modify our ideas from [14] and use a construction tree of simplices; we present two approaches, one based on Euclidean tetrahedra, the other on spherical triangles. Both approaches reveal close structural similarities between the kinematics of single-vertex folds and the kinematics of Euclidean planar loops.

\section{A. Approach One: Trees of Euclidean Tetrahedra}

System Modeling. Pick a non-apex point $P(i)$ on the $i^{\text {th }}$ crease line (in cyclic order, with $i$ taken modulo $n$ as needed). The line segment $\overline{P(i) P(i+1)}$ is contained in the $i^{\text {th }}$ panel. The panels are rigid and rotation around a crease line is a rigid motion, so the length $l_{i}$ of this segment is constant over all deformations; thus taken together all these segments form a (spatial) loop of $n$ rigid links. Moreover, the length of $\overline{O P(i)}$ (on the $i^{\text {th }}$ crease line) is also constant, for the same reason. Thus each triangle $\operatorname{Tr} i(O, P(i), P(i+1))$ maintains its congruence class throughout all deformations of the generalized cone, so from the point of view of deformation space the original generalized cone may be replaced with a polyhedral cone comprising the $n$ triangular panels $\operatorname{Tr} i(O, P(i), P(i+$ $1)), i=0, \ldots, n-1$. We now find a construction tree of simplices for this cone, and use simplex-based parameters to study its DSpace.

Tetrahedra Trees and Parameters. One method is to use any construction tree of triangles for the loop $(P(0), P(1), \ldots, P(n-1))$. For example, a 6-bar loop with vertices $P(0), \ldots, P(5)$ is decomposed by the three diagonals $(P(1), P(3)),(P(3), P(5))$, and $(P(5), P(1))$ into a construction tree of 4 triangles: Tri $(1,3,5)$, Tri $(1,2,3)$, Tri $(3,4,5)$, and $\operatorname{Tr} i(5,0,1)$, with $\operatorname{Tr} i(1,3,5)$ adjacent to all other three. (We abbreviate $\operatorname{Tri}(P(1), P(2), P(3))$ by $\operatorname{Tr} i(1,2,3)$ and so on.) Now, each of these triangles $\operatorname{Tr} i(P(i), P(j), P(k))$ determines a tetrahedron $\operatorname{Tet}(O, P(i), P(j), P(k))$, whose three edges (additional to those of the triangle) have already been noted to be of fixed length. These tetrahedra fit together, in a tree combinatorially identical to that chosen to construct the loop, so as to construct the polyhedral cone (see Fig. 2.) The corresponding tetrahedra-based parameters for DSpace of the cone are closely related, but not identical, to the triangle-based

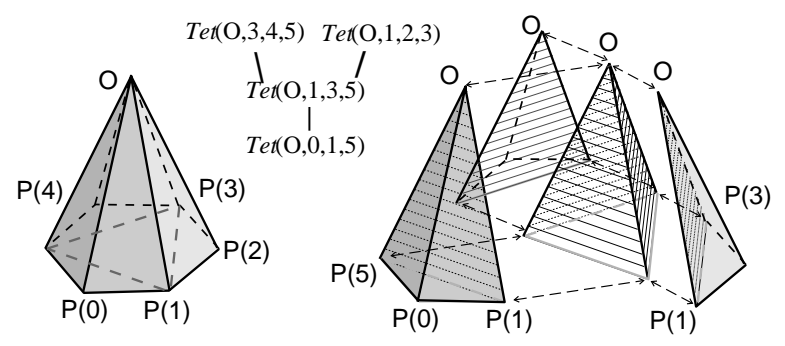

Fig. 2. Decomposition of a polyhedral cone into a tree of tetrahedra. 
parameters for DSpace of the loop. Namely, we use (a) the squared diagonal lengths $D[\mathcal{T}]=[D[\mathcal{T}](1), \ldots, D[\mathcal{T}](n-3)]$, and (b) the tetrahedron orientation signs $s[\mathcal{T}]=[s[\mathcal{T}](1), \ldots$, $s[\mathcal{T}](n-2)]$. Here $\mathcal{T}$ denotes a construction tree of tetrahedra for the polyhedral cone, $D[\mathcal{T}](i)=d^{2}[\mathcal{T}](i)$ is the squared length of the $i^{\text {th }}$ diagonal, and $s[\mathcal{T}](j)$ is the orientation sign of the $j^{\text {th }}$ tetrahedron comprised in $\mathcal{T}$. (The number $n-3$ of squared diagonal lengths for the tree of tetrahedra equals the number $n-3$ of diagonal lengths for the tree of triangles, and similarly for the number $n-2$ of orientation signs.)

Constraints and DSpace Structures in Tetrahedral Parameters. In this formulation, the loop closure constraint on the panels of our polyhedral cone becomes that set of constraints on the diagonal lengths which is necessary and sufficient for the tetrahedra comprised in $\mathcal{T}$ to exist. These constraints can be phrased in terms of the Cayley-Menger determinant. As is well known, given real numbers $D(i, j)(0 \leq i, j \leq 3)$ with $D(i, j)=D(j, i) \geq 0, D(i, i)=0$, points $P(i) \in \mathbb{R}^{3}$ $(0 \leq i \leq 3)$ with $D(i, j)=\|P(j)-P(i)\|^{2}$ exist if and only if

$$
\left|\begin{array}{ccccc}
0 & 1 & 1 & 1 & 1 \\
1 & 0 & D(0,1) & D(0,2) & D(0,3) \\
1 & D(1,0) & 0 & D(1,2) & D(2,3) \\
1 & D(2,0) & D(2,1) & 0 & D(2,3) \\
1 & D(3,0) & D(3,1) & D(3,2) & 0
\end{array}\right| \geq 0 .
$$

Equality holds if and only if the points $P(i)$ are coplanar. Each of the $n-2$ tetrahedra $\sigma \in V(\mathcal{T})$ gives us one such inequality constraint. The set of all $n-2$ inequalities defines the set of feasible squared diagonal lengths for the $n$-sided polyhedral cone, which we call DStretch.

The tetrahedron orientation signs are likewise defined along the lines set out in [14] for triangle orientation signs for a loop with a construction tree of triangles. If we use + and - for the two orientations of non-degenerate tetrahedra, and label singular tetrahedra with both + and -, we obtain (roughly) an identification of DSpace $[\mathcal{T}]$ of the polyhedral cone parametrized by the tree $\mathcal{T}$ of tetrahedra with DStretch $[\mathcal{T}] \times D F l i p$, where DFlip $=\{+,-\}^{n-2}$. (As in [14], eliminating the "roughness" requires analysis of "super-singular" deformations.) Such a DSpace parametrization is very similar to that for a planar loop with $n$ links given in [14]. An important difference is that, whereas [14] proves that DStretch of a planar loop with $n$ revolute joints and fixed link lengths is a convex polyhedron of dimension $n-3$, in the present case DStretch is still convex but (for $n \geq 5$ ) it is no longer a polyhedron.

The geometry of its various curvilinear faces is revealed by separate analyses of the constraint (2) in the cases where the corresponding tetrahedron includes exactly 1,2 , or 3 diagonals: a single diagonal's squared length is constrained to lie in an interval; a pair of diagonals' squared lengths are constrained to lie inside or on a certain ellipse; and a triple of diagonals' squared lengths lie inside or on the boundary of a certain semi-algebraic (cubic) convex body.

\section{B. Approach Two: Trees of Spherical Triangles}

For the tetrahedra above, the non-apex points $P(i)$ on the crease lines, and thus their distances to the cone apex $O$ and the link lengths, can be chosen quite arbitrarily, though astute choices might afford extra convenience in computation.

System Modeling. For the second approach, we demand that all $P(i)$ 's be at the same distance from the cone apex $O$. Thus the set of $P(i)$ 's is the intersection of the crease lines with a sphere centered at $O$. As noted earlier, the distance between $O$ and any point on a crease line is constant across all deformations, so this sphere is also constant; further, since the cone panels are assumed to be rigid and planar, each of them intersects the sphere in a spherical line segment (i.e., an arc of a great circle through the corresponding points $P(i)$ and $P(i+1)$ ), and the spherical length of that segment is also constant. For simplicity, instead of spherical length we use central angle to measure spherical line segments (the spherical length is the radius of the sphere multiplied by the radian measure of the central angle). Following standard usage from spherical geometry, we also call a spherical line segment minor if its central angle is in $[0, \pi]$, and major if not.

In this language, what we have observed is that the intersection of each cone panel intersects the sphere in a spherical line segment of constant central angle. Therefore the intersection of the sphere with the entire system of rigid and planar panels incident on the cone apex $O$ is a spherical $n$-gon loop ( $n$ being the number of panels) with links of fixed central angles; and the kinematics of the single-vertex fold is equivalent, in the sense of having identical DSpaces, to that of this spherical loop. The converse, that every spherical loop corresponds in this way to a single-vertex fold, is obvious.

There is a subtlety which we have no room to discuss properly here, but which must be mentioned. It is entirely standard to define the spherical distance between two points of a sphere to be the length of a minor spherical line segment with those endpoints, and with that definition the sphere becomes a metric space in the usual way-in particular, the triangle inequality holds; but there is no compelling reason to require the edges of a spherical polygonal $n$-gon to be minor segments. Nonetheless, in the remainder of this paper we restrict our attention to spherical polygonal $n$-gon loops in which each edge is minor; equivalently, in the language of foldable objects, to single-vertex folds in which the angle of each panel at the cone apex is at most $\pi$.

Spherical geometry shares many theorems with Euclidean plane geometry, but not all; so our triangle approach to spherical $n$-gon loop kinematics is very similar, but not identical, to our triangle approach in [14] for Euclidean planar $n$-gon loops. Below, we emphasize the points of difference.

\section{Spherical Triangle Trees and Parameters.}

The construction trees of triangles in [14] have no branching because the triangles are anchored (share a common vertex); however, as noted in the proof of Theorem 1, the results and arguments in [14] extend to arbitrary construction trees of triangles, Euclidean or spherical. In particular, for an $n$-gon 
loop each construction tree has $n-2$ nodes and $n-3$ edges (loop diagonals); Fig. 3 shows this for a spherical 6-gon with central angles $(50,97,151,82,35,59)$ (in degrees).

Following our general simplex-based approach, we define spherical-triangle-based parameters for the deformations of a spherical loop, and thus that of a polyhedral cone allowing rotation about the edges. Namely, we use (a) the spherical diagonal lengths $\beta[\mathcal{T}](1), \ldots, \beta[\mathcal{T}](n-3)$, and (b) the spherical triangle orientation signs $s[\mathcal{T}](1), \ldots, s[\mathcal{T}](n-2)$, where $\mathcal{T}$ is a construction tree of spherical triangles for the spherical loop, $\beta[\mathcal{T}](i)$ is the central angle of diagonal $i$, and $s[\mathcal{T}](i)$ is the orientation sign $(+,-$, or 0$)$ of the $i^{t h}$ spherical triangle in $\mathcal{T}$. One way to define this sign is as the orientation sign of the tetrahedron formed by the triangle vertices (in their given order) preceded by the center of the sphere; as usual 0 means that the tetrahedron is degenerate (equivalently, that the triangle is contained in a great circle). Reasoning like that in [14] shows that the orientation sign values $s[\mathcal{T}](i)$ are essentially uncoupled from the central angle parameters $\beta[\mathcal{T}](j)$; reversing the sign of a non-degenerate triangle corresponds to reversing its orientation (compare Figs. 3(a) and 4(c)).

Constraints and DSpace Structures in Spherical Triangle Parameters. To generalize the method given in [14], we need to find the necessary and sufficient conditions on three angles less than or equal to $\pi$ such that there exist a spherical triangle with those central angles. This is easy to do in light of the observation (true in both spherical and Euclidean geometry) that the length of one edge of a triangle is at most the sum of the lengths of the other two edges. One issue here is that the spherical distance between any two points is restricted to $[0, \pi]$. Given two distances $\beta_{1}, \beta_{2}$, each between 0 and $\pi$, their usual sum $\beta_{1}+\beta_{2}$ will have a direct range of $[0,2 \pi]$. However, if we define their spherical sum by

$$
\beta_{1} \oplus \beta_{2}=\min \left(\beta_{1}+\beta_{2}, 2 \pi-\beta_{1}+\beta_{2}\right)
$$

then we find that their spherical sum is again between 0 and $\pi$; and we see that given a spherical triangle with vertices $P(i)$, $P(j), P(k)$ at spherical distances $\beta(i, j), \beta(j, k)$ and $\beta(k, i)$ in $[0, \pi]$, the triangle inequalities become

$$
\begin{aligned}
& \beta(i, j) \leq \beta(j, k) \oplus \beta(k, i) \\
& \beta(j, k) \leq \beta(k, i) \oplus \beta(i, j) \\
& \beta(k, i) \leq \beta(i, j) \oplus \beta(j, k)
\end{aligned}
$$

Moreover, in the cube $[0, \pi]^{3}$, these inequalities (which are all linear in the coordinates) cut out a convex polyhedron.

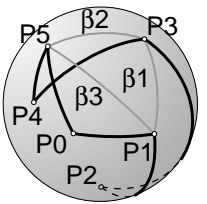

(a)

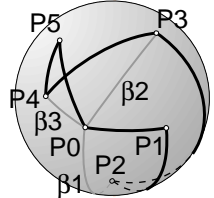

(b)

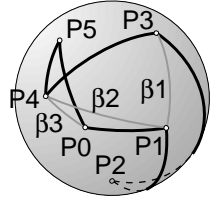

(c)
Fig. 3. One deformation of a spherical 6-gon loop with 3 sets of shape parameters derived from 3 different trees of 2 -simplices.

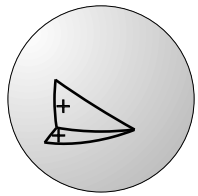

(a) Like.

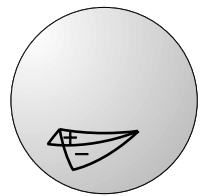

(b) Unlike

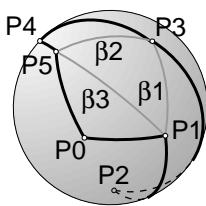

(c) Cf. Fig. 3(a)
Fig. 4. (a), (b) Two embeddings, with different relative orientations, of one pair of spherical triangles. (c) A deformation of a 6-gon loop, differing in the orientation of exactly one triangle from that shown in Fig. 3(a).

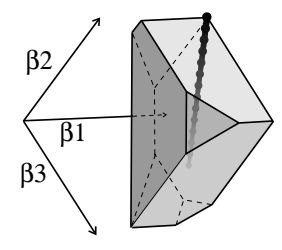

(a)

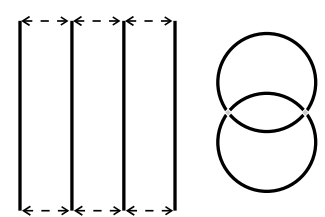

(b)
Fig. 5. (a) DStretch for a flat origami vertex with six creases and cone angles $(45 ; 30 ; 65 ; 50 ; 70 ; 100)$ degrees, and a linear path of shape parameter values joining a gray interior point $(60,60,80)$ to a black corner $(95,120,145)$. (b) DSpace for a flat origami vertex with four creases; it is not a manifold.

Now, returning to spherical loops, we note that the loop closure constraint is the set of triangle inequality constraints on the diagonal lengths required for successful formation of all spherical triangles in the construction tree $\mathcal{T}$. As in [14] this implies that DStretch, the set of feasible diagonal central angles, is a convex polytope with a natural stratification. (Fig. 5(a) illustrates the situation for a spherical 6-gon loop with central angles of $(45,30,65,50,70,100)$ degrees.)

Again as in [14], this construction endows DSpace with a stratification: roughly, DSpace is constructed by gluing together $2^{n-2}$ copies of DStretch (one for each assignment of orientation signs to triangles in $\mathcal{T}$ ) along boundary strata corresponding to singular deformations (with one or more singular triangles). One essential fact used here is that reversing the orientation of a non-singular triangle, if possible, requires passing through a singular deformation of the triangle.

As a simple example of DSpace structures, consider a flat origami vertex with 4 panels. Let its cone angles be $\beta(0,1)$, $\beta(1,2), \beta(2,3)=\pi-\beta(0,1)$, and $\beta(3,0)=\pi-\beta(1,2)$ (here we use properties (a) and (b) of flat origami vertices as quoted in section III). As the only shape parameter, we use the central angle between $P(0)$ and $P(2)$. It is easy to verify that $D$ Stretch is the interval with endpoints $|\beta(0,1)-\beta(1,2)|$ and $\beta(0,1) \oplus \beta(1,2)$. Further, at these endpoints the two triangles of the tree, one with vertices $P(0), P(1), P(2)$ and the other with vertices $P(2), P(3), P(0)$, are simultaneously singular. (Trying some concrete values for $\beta(0,1)$ and $\beta(1,2)$ may help understand this fact.) Thus when DSpace is constructed from 4 copies of the interval DStretch, at each end of the interval all four copies are glued together; the resulting DSpace is singular (i.e., not a manifold), and looks like the union of 2 circles intersecting at 2 points, as shown in Fig. 5(b). We see that all flat origami vertices of 4 panels (with generic cone angles) have topologically identical DSpaces. 


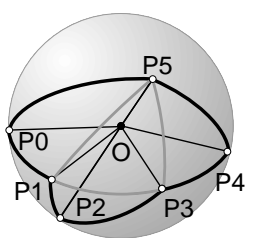

(a)

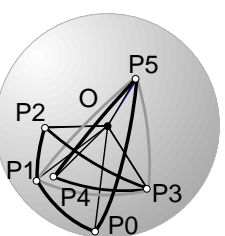

(b)

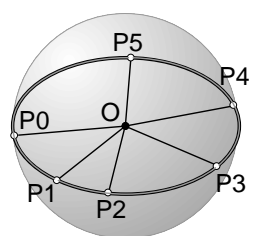

(c)

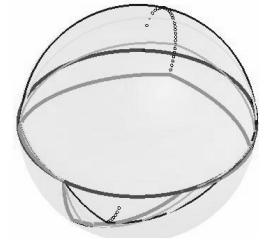

(d)

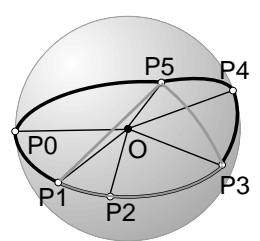

(e)

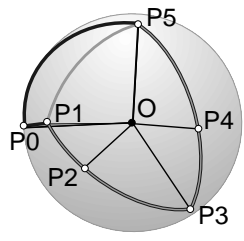

(f)

Fig. 6. Deformations and motion of the flat origami vertex with DStretch illustrated in Fig. 5(a); diagonal angles (in degrees) and orientation signs are as indicated. (a) Query deformation $1((60,60,80),(-,-,-,+))$; (b) query deformation $2((60,60,80),(+,+,+,+))$; $(\mathrm{c})$ a flat deformation $((95,120,145),(0,0,0,+))$; (d) a linear path between query deformation 1 and the flat deformation (also shown in the copy in DSpace of Fig. 5(a) that has orientation sign $(-,-,-,+))$; (e) singular deformation $1((95,60,80),(0,-,-,+))$; (f) singular deformation $2((95,120,80),(0,0,-,+))$.

\section{Results and Discussions}

The tetrahedron and spherical-triangle approaches exhibit similarity in DSpace structures for single-vertex fold kinematics, as summarized in the following theorem.

Theorem 2: For a single-vertex fold with $n$ rigid panels, DStretch is an $(n-3)$-dimensional convex body, DFlip is the discrete set $\{+,-\}^{n-2}$, and DSpace consists of $2^{n-2}$ copies of DStretch properly identified along their boundaries.

Both approaches have significant advantages over approaches that use joint angle parameters, thanks both to the explicit constraints in our new parameters and the practical convexity of DSpace in these parameters. As an illuminating example for the efficiency of our simplex-based approach, consider the generation of loop deformations. In our new parameters, this can be done in two steps.

First, we solve the shape parameters. Both Cayley-Menger determinant and spherical triangle inequality constraints can be efficiently solved, e.g., with convex programming (or, for triangle inequalities, linear programming). We have also developed our own solving methods that take advantage of the highly structured constraints; in our computation study using Matlab, they beat the general methods by orders of magnitude.

Second, we pair the shape parameter values with the relative orientation sign parameters. For a generic single-vertex fold with $n$ rigid panels, each set of diagonal length parameter values of a non-singular deformation pairs with $2^{n-2}$ distinct sets relative orientation signs, generating $2^{n-2}$ distinct (though related) system deformations, all with the same simplex shapes but differing simplex orientations. On a laptop computer, using linear programming in Matlab, a set of valid shape parameters for a generic single-vertex 1000-panel fold can be generated in about 1 millisecond. Paired with the points of DFlip, this yields $2^{998}$ different deformations.

Knowledge and nice geometry of system DSpaces have significant impact on many kinematics related issues such as system design and path planning. For example, it is foreseeable for certain design tasks to favor systems in which having all (or important) system configurations fall into a single connected component of DSpace (or of DFree, when collision avoidance is taken into consideration). Explicit and efficient parametrization of DSpaces provides invaluable tools to designers for the evaluation and improvements of design schemes. As another example of the broad implications of our approach and results, the piecewise convexity of DSpace in simplex-based parameters greatly simplifies path planning for single-vertex folds. That is, two query deformations in the closure of one DSpace stratum (roughly, the points of DSpace corresponding to one copy of DStretch) can be joined by a straight-line path because DStretch is convex. Two deformations merely in the same component of DSpace can be joined by a piecewiselinear path, once we determine critical singular deformations through which to move successively between strata; again, the simple nature of the constraints on our shape parameters leads to efficient computation of singular deformations.

Figs. 6(a) and 6(b) show two query deformations of a flat origami vertex with 6 panels, again having central angles of $(45,30,65,50,70,100)$ degrees: both deformations have diagonal length values of $(60,60,80)$ degrees, and have opposite orientations for all triangles except the one with vertices $P(1)$, $P(3), P(5)$. Each query deformation can be linearly connected to a flat deformation, like that in Fig. 6(c) with diagonal lengths of $(95,120,145)$ degrees. Fig. 6(d) illustrates a linear path from the deformation in Fig. 6(a) to the flat deformation. While a linear path between two points in a convex set is shortest in the convex metric and mathematically optimal, it generally involves simultaneous motion (folding) of all crease lines. Since in practice many folding motions are most easily done by folding only one or a few creases at once, for folding problems Manhattan paths are likely to be more practical than linear paths. The query deformation in Fig. 6(a) can reach the flat deformation by using a 3 -segment Manhattan path through two singular deformations as in Figs. 6(e) and 6(f).

For single-vertex fold systems, the spherical triangle approach will generally be more efficient than the tetrahedron approach, since triangle constraints (inequalities (4)-(6)) are generally simpler than determinant constraints (inequality (2)). We introduced both approaches since each has its own advantages in various situations; e.g., the tetrahedron approach generalizes directly to multi-vertex folds (like that in Fig. 7)

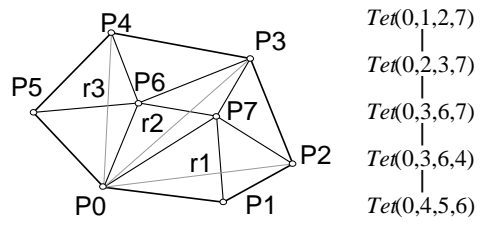

Fig. 7. A multi-vertex fold with a construction tree of simplices. 
that allow construction trees of tetrahedra. Note that a multivertex fold contains multiple loops of panels; Fig. 7 is just one of the simplest examples of a multi-loop, multi-point system allowing a construction tree of simplices. Our approach and results, described in section II and illustrated in this section for single-vertex folds, apply directly to all such systems.

\section{SUMmary}

In this paper, we have described our novel simplex-tree based approach for the kinematics of multi-body systems involving loops, and illustrated it with an example system of single-vertex folds. For systems allowing construction trees of simplices, we efficiently use simplex-based shape and orientation parameters for system deformations, formulate loop constraints as constraints on shape parameters needed for successful formation of the simplices (e.g., the triangle inequality and Cayley-Menger determinant constraints for triangles and tetrahedra), solve the set of valid length parameters, and explicitly construct the DSpace structures essentially as the product of the shape parameter set and the orientation parameter set (which carries no loop constraints). For systems involving loops, knowledge of DSpace and CSpace structures with explicit parametrizations is invaluable for many kinematics related issues, e.g., motion planning, system design, analysis, simulation, and control; until recently ( $c f .[7,8,14$, 15]) these structures and parametrizations, and techniques for working with them, have largely remained elusive. In this paper we have greatly extended our work in $[14,15]$.

Part of our ongoing research is to identify and study systems allowing simplicial construction trees. For a multipoint linkage system (again, with links modeling distance constraints), the conditions on the existence of a construction tree can be intuitively understood as follows: its constraints as reflected in the existing links shall allow the addition of virtual links that decompose the system into a collection of simplices (or more broadly, a collection of simplices with other rigid system components, as dictated by existing system constraints, attached to the simplices) with edges consisting of all existing and virtual links, and with free relative configurations allowed among adjacent simplices. Lengths of virtual links and existing links with non-trivial ranges are captured by shape parameters, relative configurations by orientation parameters. We are currently working on further properties and algorithms useful to identify these systems and their parameters. We emphasize that, while the representative systems solved in simplex-tree based approaches so far (including Euclidean polygonal loops in our earlier papers $[14,15]$ and the systems in this paper) use construction trees with equidimensional simplices as nodes, our simplex-based approach allows non-equidimensional simplices as nodes of a single tree. In future papers we will describe practical robotic systems that can be usefully studied using construction trees of non-equidimensional simplices.

\section{ACKNOWLEDGEMENT}

We thank the reviewers and area chairs for help and advice. We were supported in part by NSF Grant IIS-0713335.

\section{REFERENCES}

[1] J. J. Craig, Introduction to Robotics: Mechanics and Control, 2nd Edition. Reading, MA: Addison-Wesley Publishing Company, 1989.

[2] R. M. Murray, Z. Li, and S. S. Sastry, A Mathematical Introduction to Robotic Manipulation. Boca Raton, FL: CRC Press, 1994.

[3] J.-P. Merlet, Parallel Robots. Springer, 2000.

[4] M. Mason, Mechanics of Robotic Manipulation. The MIT Press, 2001.

[5] M. Kapovich and J. Millson, "On the moduli spaces of polygons in the euclidean plane," J. Diff. Geom., vol. 42, pp. 133-164, 1995.

[6] _ - "On the moduli space of a spherical polygonal linkage," Canadian Mathematical Bulletin, vol. 42, pp. 307-320, 1999.

[7] R. Milgram and J. Trinkle, "The geometry of configuration spaces for closed chains in two and three dimensions," Homology Homotopy and Applications, 2002.

[8] J. Trinkle and R. Milgram, "Complete path planning for closed kinematic chains with spherical joints," Int. J. Robot. Res., vol. 21, no. 9, pp. 773789, 2002

[9] J. Porta, L. Ros, and F. Thomas, "Inverse kinematics by distance matrix completion," Proc. of 12th International Workshop on Computational Kinematics, 2005.

[10] — " "On the trilaterable six-degree-of-freedom parallel and serial manipulators," in Proc. IEEE Int. Conf. Robot. Autom. (ICRA), 2005

[11] _ "Multiple-loop position analysis via iterated linear programming," Robotics: Science and Systems, 2006.

[12] C. Torras, F. Thomas, and M. Alberich, "Stratifying the singularity loci of a class of parallel manipulators," IEEE Trans. Robot., pp. 23-32, 2006.

[13] M. Alberich, F. Thomas, and C. Torras, "Flagged parallel manipulators," IEEE Trans. Robot., pp. 1013-1023, 2007.

[14] L. Han, L. Rudolph, J. Blumenthal, and I. Valodzin, "Convexly stratified deformation spaces and efficient path planning for planar closed chains with revolute joints," in Proc. Seventh International Workshop on Algorithmic Foundation of Robotics, 2006.

[15] L. Han and L. Rudolph, "Inverse kinematics for a serial chain with joints under distance constraints," in Robotics: Science and Systems (RSS), 2006.

[16] — "A unified geometric approach to inverse kinematics of a spatial chain with spherical joints," 2006, accepted to ICRA 2007.

[17] L. M. Blumenthal, Theory and Applications of Distance Geometry, 2nd edition. American Mathematical Society, 1970.

[18] D. Balkcom and M. Mason, "Introducing robotic origami folding," in Proc. IEEE Int. Conf. Robot. Autom. (ICRA), 2004.

[19] D. J. Balkcom, E. Demaine, and M. Demaine, "Folding paper bags," in Annual Workshop on Computational Geometry, 2004.

[20] S. Gupta, D. Bourne, K. K. Kim, and S. S. Krishnan, "Automated process planning for robotic sheet metal bending operations," Journal of Manufacturing Systems, 1998.

[21] L. Lu and S. Akella, "Folding cartons with fixtures: A motion planning approach," IEEE Trans. Robot. Automat., pp. 346-356, 2000.

[22] G. Song and N. M. Amato, "A motion planning approach to folding: From paper craft to protein folding," in Proc. IEEE Int. Conf. Robot. Autom. (ICRA), 2001, pp. 948-953.

[23] J. Liu and J. Dai, "An approach to carton-folding trajectory planning using dual robotic fingers," Robotics and Autonomous Systems, vol. 42, pp. $47-63,2003$.

[24] T. C. Hull, "On the mathematics of flat origamis," Congressus Numerantium, vol. 100, pp. 215-224, 1994.

[25] — "The combinatorics of flat folds: a survey," in Proc. 3rd International Meeting of Origami Science, Math, and Education, 2001.

[26] E. D. Demaine and M. L. Demaine, "Recent results in computational origami," in Proc. 3rd International Meeting of Origami Science, Math, and Education, 2001.

[27] I. Streinu and W. Whiteley, "Single-vertex origami and spherical expansive motions," in Proc. Japan Conf. on Discrete and Comp. Geometry Tokai University, Tokyo, Oct. 8-11 2004. Lecture Notes in Computer Science 3742, pp. 161-173, Springer 2005., 2004.

[28] R. Connelly, E. D. Demaine, and G. Rote, "Straightening polygonal arcs and convexifying polygonal circles," Discrete and Computational Geometry, 2003.

[29] I. Streinu, "A combinatorial approach to planar non-colliding robot arm motion planning," in Proc. IEEE Symp. Foundations of Computer Science (FOCS), 2000, pp. 443-453. 